\title{
Evaluation of Factors Determining Tracheostomy Decannulation Failure Rate in Adults: An Indian Perspective Descriptive Study
}

\author{
Tapasya Bishnoi $^{1}$ (D) Pankaj Kumar Sahu ${ }^{1}$ - A. P. Arjun ${ }^{1}$
}

Received: 13 June 2020/ Accepted: 16 July 2020/Published online: 24 July 2020

(C) Association of Otolaryngologists of India 2020

\begin{abstract}
Decannulation is an essential step in liberating tracheostomised patients from mechanical ventilation. This procedure is purely based on the clinician's judgment and there is no universally accepted protocol to date for this vital procedure. This study aimed to describe decannulation practice and failure rates in patients with tracheostomy and to determine the factors associated with the outcome of tube removal. A prospective study was done on 50 patients (both sexes) who required a tracheostomy and cared for at Command Hospital Bangalore Center between January 2019 and April 2020. Data were analyzed using descriptive and inferential tests. Out of the 50 decannulation decisions, 7 patients experienced decannulation failures giving a failure rate of $14 \%$. Out of the 7 decannulation failure cases, about 4 patients (10\%) experienced difficulty in swallowing and 3 patients (2\%) experienced stridor. There was no associated mortality. A decannulation failure of $14 \%$ was seen in this study in tracheostomised patients after prolonged mechanical ventilation. Various factors govern the success of tracheostomy decannulation procedures which occur during the first 24-48 h after decannulation. Lack of swallowing/secretions/cough management and the development of stridor were the commonest cause of decannulation failure in this study.
\end{abstract}

Tapasya Bishnoi

Tapasya26bishnoi@gmail.com

Pankaj Kumar Sahu

drpksahu0711@gmail.com

A. P. Arjun

Arjun.mmc@gmail.com

1 ENT, Command Hospital Air Force Bengaluru, Bangalore, India
Keywords Tracheostomy · Decannulation failure · Ventilation

\section{Introduction}

The first known depiction of tracheostomy was from 3600 BC, on Egyptian tablets [1]. According to legend, Alexander the Great used his sword to open the airway of a soldier choking from a bone lodged in his throat [2]. Despite its antiquity, tracheostomy was rarely performed until the beginning of the nineteenth century and until less than 20 years ago its use was limited to surprisingly few conditions. At present, tracheostomy is more commonly used for prolonged mechanical ventilation rather than for upper airway obstruction.

Prolonged tracheostomy tube placement may expose the patients to an increased risk of late complications, including tracheal stenoses, bleeding, fistulas, infections, and aspiration [3]. Psychological implications are profound with patients experiencing reduced body image perceptions and life satisfaction. Removing a tracheostomy is a fundamental step in rehabilitating a patient recovering from critical illness. This process of weaning from tracheostomy to maintenance of spontaneous respiration and/or Tracheostomy is a simple, life-saving procedure done to establish airway in-patients with acute respiratory failure with the expected need for prolonged mechanical ventilation, failure to wean from mechanical ventilation, upper airway obstruction, difficult airway, and copious secretions [4]. It requires an opening to be made in the anterior wall of the trachea and a tube is inserted through the opening to allow passage of air and removal of secretions. This allows the patients to breathe through the tracheostomy tube, instead of the nose and mouth. Approximately $10 \%$ of 
mechanically ventilated critically ill patients receive a tracheostomy to facilitate prolonged airway and ventilatory support [5].

Airway protection is termed "decannulation". This simple step requires near-perfect coordination of the brain, swallowing, coughing, phonation, and respiratory muscles [6]. However, multifactorial aberrations in this complex interplay can result in its failure. There are many advantages to decannulation, including improved phonation and swallowing function. Discharging patients home or to another care facility is an easier process if the patient or their caregivers do not need to learn how to manage a tracheostomy tube. Also, decannulation improves patient comfort and perceived physical appearance [7].

Little evidence is available to guide the process of weaning and optimal timing of tracheostomy tube removal. Thus, decannulation decisions are based on clinical judgment [8]. The incidence of decannulation failures is reported to be between 2 and $25 \%$ of decannulation attempts [7, 9-12]. Decannulation failure is associated with unfavorable outcome [6].

Hence, the objectives of the study were: (1) To describe the decannulation failure rate during the rehabilitative process following tracheostomy in patients in a tertiary hospital and (2) to identify the factors associated with the outcome of tracheostomy tube removal.

\section{Materials and Methods}

This was a prospective descriptive study of 50 tracheostomised patients carried out from January 2019 to April 2020, at Command Hospital Bangalore. The study was approved by the Institutional Ethical Committee. Informed consent was obtained from all the participants in the study. Patients included in this study were from Surgery Ward, Trauma ICU, and Casualty. The age range varied between 18 and 85 years. The inclusion criteria were patients who had tracheostomy procedure on an emergency basis where prolonged mechanical intubation was difficult or not possible, patients who underwent elective tracheostomy where prolonged mechanical intubation and stridor were anticipated and tracheostomy was done in intubated patients. All patients with tracheostomy were included in the present study. Detail history of patients such as age, sex, and occupation, economic status of the patient address, and symptoms of the patients was noted.

Initially, all the basic details regarding the type of injury and nature of the injury, surgical intervention, the indication, and the type of tracheostomy were endorsed in a proforma.

Then the patients were assessed for resolution of the primary cause of tracheostomy, if yes then primary reflexes were assessed like cough, swallow, and gag. In addition to this FOL (fiberoptic laryngoscopy) was done to know about vocal cord status and glottic chink and a tracheal aspirate swab was taken and sent for microbiological evaluation. Besides, a chest $\mathrm{x}$-ray was done to rule out any pulmonary pathology. If all the assessed parameters were within the normal limits, then the decannulation trial was proceeded by downsizing the tracheostomy tube.

\section{Decannulation Procedure}

A synchronized cuff-deflation technique was used every time the cuff was deflated and required two competent practitioners. As the cuff was deflated using a $10 \mathrm{ml}$ syringe, suction was given via the tracheostomy tube to remove any secretions that come from above the cuff into the trachea. If the patient had a tracheostomy tube with a subglottic port, it had to be aspirated before the cuff deflation to remove secretions above the cuff. When a standard cuffed tube was used, oral suction was performed before cuff deflation. Continuous monitoring of oxygen saturation levels was essential, as these may fall during the weaning process. Oxygen saturation levels were maintained at the target levels. Oxygen was administered if required at the prescribed rate [9].

Cough effectiveness, swallowing and gag reflex, quantity, and quality of secretions, duration of mechanical ventilation, sepsis, etiology of respiratory failure, and comorbidities were the criteria used for predicting successful decannulation. Principal parameters considered were the patient's ability to remove secretions, swallowing function, absence of psychiatric diseases, the possibility of reaching spontaneous breathing, and the amount of respiratory space.

1. The presence and efficacy of voluntary cough were assessed based on a study by Bach et al. [9], in which subjects were evaluated with a spirometer. Subjects had a negative score when cough peak flow $<160$ $\mathrm{L} / \mathrm{min}$ or they could not cough voluntarily due to cognitive deficits.

2. Tracheostomy tube capping for at least $72 \mathrm{~h}$. This consisted of the ability to keep the tracheostomy stoma closed and breathe through the mouth. This has been described by Stelfox et al. [10] as a fundamental determinant for tracheostomy decannulation.

3. Swallowing instrumental assessment was carried out through fibro-endoscopic evaluation with liquid and food administrations, reported as the accepted standard for swallowing [11]. Severity was assessed using the penetration aspiration scale (scores 1-8) [12]. A score of 1 indicates the absence of dysphagia, scores 
between 2 and 5 indicate swallowing difficulties and penetration events, and scores $>5$ show aspiration events.

4. The number of tracheal suctions were recorded. A negative score was considered as 2 or more suctions every $8 \mathrm{~h} \mathrm{[13].}$

5. Instrumental evaluation of the patency of the airways was evaluated by endoscopy, considering tracheal stenosis as lumen diameter $<50 \%[14]$.

6. $\mathrm{S}_{\mathrm{pO} 2}>95 \%$ in ambient air [15].

\section{Statistical Methods}

Decannulation failure was considered as the primary outcome variable. Gender, Surgical intervention, a period of ventilation, mode of ventilation, indication of tracheostomy, percutaneous/elective/emergency, tracheostomy in situ duration, duration of TT change, swallowing, GAG, associated comorbidity and hospital stay in duration were considered as a primary explanatory variable. Descriptive analysis was carried out by mean and standard deviation for quantitative variables, frequency, and proportion for categorical variables. Univariate binary logistic regression analysis was performed to test the association between the explanatory variables and outcome variables. Unadjusted odds ratio along with $95 \%$ CI was presented. Variables with statistical significance in univariate analysis were used to compute multivariate regression analysis. The adjusted odds ratio along with their $95 \%$ CI was presented. $P$ value $<0.05$ was considered statistically significant. IBM SPSS version 22 was used for statistical analysis [16].

\section{Results}

A total of 50 subjects were included in the final analysis.

Among the study population, the majority of 40 $(80.00 \%)$ were male. Among the study participants, 9 (18.00\%) had smoking as a habit and 39(78\%) had no habits at all. Among the study participants with primary diagnosis, $31(62.00 \%)$ were severe head injury, 8 $(16.00 \%)$ was head injury with polytrauma, $3(6.00 \%)$ were meningitis/meningoencephalitis and stroke in each, 2 $(4.00 \%)$ were meningioma, $1(2.00 \%)$ were cerebral malaria, cortical venous thrombosis and Ludwig's angina each (Table 1).

Among the study participants, $29(58.00 \%)$ went with Surgical Intervention. the mean of the period of ventilation (days) was $5.78 \pm 3.99$, Among the study participants majority, 49 (98.00\%) were reported mechanical mode of ventilation and. The majority of study population 44 $(88.00 \%)$ were under prolonged ventilator support, 5 $(10.00 \%)$ went with surgical intervention and only 1 $(2.00 \%)$ was reported desaturation in indication of tracheostomy. Among the study participants, 45 (90\%) were reported elective tracheostomy and $5(10 \%)$ were emergency tracheostomy. The mean of Tracheostomy in situ duration (months) was $5.36 \pm 9.93$ (Table 2).

Among the study population with swallowing, 45 $(90.00 \%)$ were reported swallowing, $38(76.00 \%)$ were with cough and $48(96.00 \%)$ were with gag. All the study population $50(100.00 \%)$ reported as phonation. The majority of the study population, $48(96.00 \%)$ were used bilateral vocal cord mobile. Among the study participants, only $3(6.00 \%)$ were Pseudomonas positive In Tracheal Aspirate. In chest X-ray, 49 (98.00\%) were showed normal and $1(2.00 \%)$ was with COPD changes. Among the decannulation failure people, $3(6.00 \%)$ were due to absent/ sluggish swallow/cough/gag reflex, $1(2.00 \%)$ was due to desaturation, and due to excessive secretions each. Among the study participants, 7 (14.00\%) reported as Dec annulation failure (Table 3).

The univariate logistic regression analysis had shown a statistically less significant association with decannulation failure with all explanatory factors as presented in the table $(P$ value $>0.05)$ (Table 4$)$. These insignificantly independent variables, or in combination with other factors show significance in predicting the success of decannulation.

\section{Discussion}

Weaning success is an outcome reported by numerous papers in the literature [17-19]. For the most part, the authors reported successful weaning from the tracheostomy in the ICU context taking into account patients with different pathologies (neurological, cardiac, pulmonary).

This study evaluated 50 tracheostomised patients of both sexes, between 18 and 85 years of age. Out of these patients, 7 experienced decannulation failure, giving a failure rate of $14 \%$ in this study. Clinicians should understand that tracheostomy decannulation is not without risk. However, there is currently no accepted definition for decannulation failure.

We defined weaning failures according to the Stelfox definition [10]. Stellfox guidelines outline that if any respiratory failure happens after $48-96 \mathrm{~h}$ from the weaning attempt, a regression to the previous condition of being tracheostomised is needed [20]. It is important to highlight that in several papers the definition for failures, unfortunately, is not univocal, ranging from $24 \mathrm{~h}$ to 1 week, while other authors define a weaning failure as when the patient 
Table 1 Descriptive analysis of Baseline parameters in the study population $(\mathrm{N}=50)$

\begin{tabular}{lc}
\hline Parameter & Summary (n\%) \\
\hline Gender & \\
Male & $40(80.00 \%)$ \\
Female & $10(20.00 \%)$ \\
Habits & \\
Alcohol & $1(2.00 \%)$ \\
Smoking & $9(18.00 \%)$ \\
Smoking/ alcohol & $1(2.00 \%)$ \\
Nil & $39(78.00 \%)$ \\
Primary diagnosis & \\
Severe head injury & $31(62.00 \%)$ \\
Head injury with polytrauma & $8(16.00 \%)$ \\
Meningitis/meningoencephalitis & $3(6.00 \%)$ \\
Stroke & $3(6.00 \%)$ \\
Meningioma & $2(4.00 \%)$ \\
Cerebral malaria & $1(2.00 \%)$ \\
Cortical venous thrombosis & $1(2.00 \%)$ \\
Ludwigs angina & $1(2.00 \%)$ \\
\hline
\end{tabular}

Table 2 Descriptive analysis of clinical parameters in the study population $(\mathrm{N}=50)$

\begin{tabular}{ll}
\hline Parameter & Summary \\
\hline Surgical intervention & $29(58.00 \%)$ \\
Yes & $21(42.00 \%)$ \\
No & $5.78 \pm 3.99(1.0,30.0)$ \\
Period of ventilation (days) & \\
Mode of ventilation & $49(98.00 \%)$ \\
Mechanical & $1(2.00 \%)$ \\
Nil & \\
Indication of tracheostomy & $1(2.00 \%)$ \\
Desaturation & $44(88.00 \%)$ \\
Prolonged ventilator support & $5(10.00 \%)$ \\
Surgical intervention & \\
Tracheostomy & $45(90 \%)$ \\
Elective & $5(10 \%)$ \\
Emergency & $5.36 \pm 9.93(0.26,66.60)$ \\
Tracheostomy in situ duration (months) &
\end{tabular}

cannot tolerate an uncuffed fenestrated tube [19]. Most clinicians give a decannulation failure rate of 2-25\% [21].

Regarding the variables influencing decannulation patients, the important associated factors are a valid cough and the presence of a spontaneous cough. In particular, authors have found that decannulation success is more likely to happen with a valid and spontaneous cough [19]. The majority of the authors acknowledge the importance of
Table 3 Descriptive analysis of baseline parameters in the study population $(\mathrm{N}=50)$

\begin{tabular}{lc}
\hline Parameter & Summary \\
\hline Swallowing & \\
Present & $45(90.00 \%)$ \\
Absent & $5(10.00 \%)$ \\
Cough & \\
Present & $38(76.00 \%)$ \\
Absent & $12(24.00 \%)$ \\
Gag & \\
Present & $48(96.00 \%)$ \\
Absent & $2(4.00 \%)$ \\
Phonation & $50(100.00 \%)$ \\
FOL findings & \\
Bilateral vocal cord mobile & $48(96.00 \%)$ \\
Left vocal cord paresis & $1(2.00 \%)$ \\
Right vocal cord paresis & $1(2.00 \%)$ \\
Tracheal aspirate & \\
Pseudomonas positive & $3(6.00 \%)$ \\
Nil & $47(94.00 \%)$ \\
X-ray chest & \\
COPD changes & \\
Normal study & $13(14.00 \%)$ \\
Main issues for Decannulation & $1(2.00 \%)$ \\
Decannulation failure due to absent/ sluggish & \\
$\quad$ swallow/cough/gag reflex & $3(9.00 \%)$ \\
Decannulation failure due to desaturation & \\
Decannulation failure due to excessive secretions & \\
Decannulation failure due to stridor & \\
Nil & \\
Decannulation failure & \\
Yes & \\
No & \\
\hline & \\
\hline
\end{tabular}

a valid cough. In particular, such authors highlight that peak cough flow (PCF) is a crucial parameter.

Secretions management is the second crucial for the tracheostomy weaning process. In particular, several authors recognize the negative impact of unsuccessful secretions management. In this study, $56 \%$ of the decannulation failures were due to a lack of secretion management. This is following the study of Hernandez et al. who found out in their study that lack of swallowing or secretion management was the main cause of decannulation failure [22].

In our study, the next reason for decannulation failure was the development of stridor (2\%). This stridor can be due to tracheomalacia. Tracheobronchomalacia is a dynamic form of central airway obstruction characterized by an expiratory decrease of $50 \%$ or more in the crosssectional area of the tracheobronchial lumen [23]. In 
Table 4 Univariate binary logistic regression analysis of influencing Parameters on decannulation failure in the study population $(\mathrm{N}=50)$

\begin{tabular}{|c|c|c|c|c|}
\hline \multirow[t]{2}{*}{ Parameter } & \multirow[t]{2}{*}{ Un adjusted odds ratio } & \multicolumn{2}{|l|}{$95 \% \mathrm{CI}$} & \multirow[t]{2}{*}{$p$ value } \\
\hline & & Lower & Upper & \\
\hline Gender (baseline $=$ female) & 1.588 & 0.169 & 14.932 & 0.686 \\
\hline Surgical intervention $($ baseline $=$ no $)(\mathrm{N}=48)$ & 4.696 & 0.518 & 42.592 & 0.169 \\
\hline Period of ventilation (days) & 0.902 & 0.633 & 1.286 & 0.569 \\
\hline \multicolumn{5}{|l|}{ Indication of tracheostomy $($ baseline $=$ surgical $)$} \\
\hline Deranged & 0.000 & 0.000 & 0.000 & 1.000 \\
\hline Prolonged & 0.192 & 0.026 & 1.445 & 0.109 \\
\hline Tracheostomy (baseline = emergency) & 0.188 & 0.025 & 1.408 & 0.104 \\
\hline Tracheostomy in situ duration (months) & 1.015 & 0.951 & 1.084 & 0.644 \\
\hline Cough $($ baseline $=$ absent $)$ & 2.062 & 0.223 & 19.087 & 0.524 \\
\hline Swallowing (baseline $=$ absent) & 0.000 & 0.000 & 0.000 & 0.999 \\
\hline Gag $($ baseline $=$ absent $)$ & 0.000 & 0.000 & 0.000 & 0.999 \\
\hline
\end{tabular}

patients who have undergone prolonged mechanical ventilation, tracheomalacia results from an ischaemic injury to the tracheal cartilages with subsequent destruction and necrosis. These patients usually present with dyspnoea weeks to months after tracheostomy decannulation. They may also present as failure to wean from mechanical ventilation in the acute setting [24].

Other reported causes of failure in various studies were related to infections: difficulty in managing them, elevated relapse, and a selection of multi-drug resistance germs, indicating a complex situation of difficult treatment. In this study, we used a standardized protocol. Based on our study results, we believe that the use of a standardized protocol is one of the key factors for decannulation success.

\section{Limitations and Recommendations}

The main limitations of the study were the low number of participants examined and the absence of a proper followup after the 6 months spent in the operative unit. Big samples are indeed a challenge in trauma cases $[5,8,17,19]$. It was not possible to do further investigation of the patient's outcome after demission, thus, some subjects could have been decannulated after a longer period without clinical problems. A longer follow-up period of more than 6 months is definitively a functional option to analyze the subject and monitor all of the subjects in more detail.

\section{Improving Decannulation Outcomes}

Firstly, we need to understand the nature of the problem. Normally the tracheostomy is done in the intensive care units under the hands of highly skilled critical care teams.
Whereas, the decannulation procedure is done long after the patients have been brought out of the intensive care unit, where bedside caregivers may lack comprehensive experience in assessing compromised airways in patients with multiple co-morbidities. A failure of expertise commonly underlies a failure of decannulation [25].

Secondly, most hospitals do not have sufficient expert personnel to develop a team for managing decannulation care. Intensivists have some of the required skills but they often do not follow patients long term outside the ICU. Unless patients are transferred to specialized ventilator weaning centers, they often recover in acute care facilities surrounded by expertise everywhere except at the bedside [26].

Apart from the patient-related factors, if the above parameters are met, the decannulation failure rates can be minimized or reduced.

\section{Conclusion}

Tracheostomy decannulation has a risk of failure with fatal consequences if not managed appropriately. Various factors govern the success of tracheostomy decannulation procedures which occur during the first 24-48 h after decannulation. It is best if highly skilled critical care teams in the hospitals take care of theses decannulated patients with utmost care to reduce the decannulation failure rates in the future.

\section{References}

1. Trachsel D, Hammer J (2006) Indications for tracheostomy in children. Paediatr Respir Rev 7(3):162-168 
2. Servillo G, Pelosi P (2016) Tracheostomy: from surgical to percutaneous techniques. In: Servillo G, Pelosi P (eds) Percutaneous tracheostomy in critically Ill patients. Springer, Cham, pp 1-4

3. Engels PT, Bagshaw SM, Meier M, Brindley PG (2009) Tracheostomy: from insertion to decannulation. Can J Surg 52(5):427-433

4. Cheung NH, Napolitano LM (2014) Tracheostomy: epidemiology, indications, timing, technique, and outcomes. Respir Care 59(6):895-915 discussion 6-9

5. Apezteguia C, Ríos F, Pezzola D (2005) Tracheostomy in patients with respiratory failure receiving mechanical ventilation: how, when, and for whom? In: Esteban A, Cook DJ, Anzueto A (eds) Evidence-based management of patients with respiratory failure. Update in intensive care medicine. Springer, Berlin, pp 121-134

6. Singh RK, Saran S, Baronia AK (2017) The practice of tracheostomy decannulation-a systematic review. J Intensive Care $5: 38$

7. O'Connor HH, White AC (2010) Tracheostomy decannulation. Respir Care 55(8):1076-1081

8. Choate K, Barbetti J, Currey J (2009) Tracheostomy decannulation failure rate following critical illness: a prospective descriptive study. Aust Crit Care 22(1):8-15

9. Bach JR, Saporito LR (1994) Indications and criteria for decannulation and transition from invasive to noninvasive longterm ventilatory support. Respir Care 39(5):515-528 discussion 29-31

10. Stelfox HT, Crimi C, Berra L, Noto A, Schmidt U, Bigatello LM et al (2008) Determinants of tracheostomy decannulation: an international survey. Crit Care 12(1):R26

11. Cameron JL, Reynolds J, Zuidema GD (1973) Aspiration in patients with tracheostomies. Surg Gynecol Obstet 136(1):68-70

12. Rosenbek JC, Robbins JA, Roecker EB, Coyle JL, Wood JL (1996) A penetration-aspiration scale. Dysphagia 11(2):93-98

13. Martinez GH, Fernandez R, Casado MS, Cuena R, Lopez-Reina $\mathrm{P}$, Zamora $\mathrm{S}$ et al (2009) Tracheostomy tube in place at intensive care unit discharge is associated with increased ward mortality. Respir Care 54(12):1644-1652

14. Ceriana P, Carlucci A, Navalesi P, Rampulla C, Delmastro M, Piaggi G et al (2003) Weaning from tracheotomy in long-term mechanically ventilated patients: feasibility of a decisional flowchart and clinical outcome. Intensive Care Med 29(5):845-848
15. Marchese S, Corrado A, Scala R, Corrao S, Ambrosino N, Intensive Care Study Group IAoHP (2010) Tracheostomy in patients with long-term mechanical ventilation: a survey. Respir Med 104(5):749-753

16. IBM Corp (2013) IBM SPSS Statistics for Windows, Version 22.0. IBM Corp, Armonk, NY

17. Christopher KL (2005) Tracheostomy decannulation. Respir Care 50(4):538-541

18. Budweiser S, Baur T, Jorres RA, Kollert F, Pfeifer M, Heinemann F (2012) Predictors of successful decannulation using a tracheostomy retainer in patients with prolonged weaning and persisting respiratory failure. Respiration 84(6):469-476

19. Perin C, Meroni R, Rega V, Braghetto G, Cerri CG (2017) Parameters influencing tracheostomy decannulation in patients undergoing rehabilitation after severe acquired brain injury (sABI). Int Arch Otorhinolaryngol 21(4):382-389

20. Stelfox HT, Hess DR, Schmidt UH (2009) A North American survey of respiratory therapist and physician tracheostomy decannulation practices. Respir Care 54(12):1658-1664

21. Rothaar RC, Epstein SK (2003) Extubation failure: magnitude of the problem, impact on outcomes, and prevention. Curr Opin Crit Care 9(1):59-66

22. Hernandez G, Ortiz R, Pedrosa A, Cuena R, Vaquero Collado C, Gonzalez Arenas P et al (2012) The indication of tracheotomy conditions the predictors of time to decannulation in critical patients. Med Intensiva 36(8):531-539

23. Sverzellati N, Rastelli A, Chetta A, Schembri V, Fasano L, Pacilli AM et al (2009) Airway malacia in chronic obstructive pulmonary disease: prevalence, morphology and relationship with emphysema, bronchiectasis and bronchial wall thickening. Eur Radiol 19(7):1669-1678

24. Epstein SK (2005) Late complications of tracheostomy. Respir Care 50(4):542-549

25. Medeiros GC, Sassi FC, Lirani-Silva C, Andrade CRF (2019) Criteria for tracheostomy decannulation: literature review. Codas 31(6):e20180228

26. Heffner JE (2008) Tracheostomy decannulation: marathons and finish lines. Crit Care 12(2):128

Publisher's Note Springer Nature remains neutral with regard to jurisdictional claims in published maps and institutional affiliations. 